\title{
PRODUCTIVIDAD LABORAL Y SALARIO REAL: RELACIONES DE CAUSALIDAD EN VENEZUELA
}

\author{
Leonardo Vera ${ }^{\mathrm{a}}$ y Juan Andrés Vera ${ }^{\mathrm{b}}$
}

Fecha de recepción: 21 de abril de 2020. Fecha de aceptación: 30 de noviembre de 2020.

$$
\text { https://doi.org/10.22201/iiec.20078951e.2021.205.69620 }
$$

\begin{abstract}
Resumen. Para la disciplina económica no ha sido fácil encontrar una relación de causalidad clara o bien establecida entre la productividad laboral y el salario real, pese a la estrecha relación estadística y los comovimientos que varios estudios reportan. Las implicaciones de política pueden ser muy diferentes dependiendo del nexo causal que exista entre ambas variables. Usando pruebas de causalidad de Granger convencionales y pruebas de causalidad en ambiente de cointegración, tanto con datos anuales, como con datos trimestrales para Venezuela, este trabajo presenta evidencia que muestra, en contraste con el enfoque ortodoxo, que la causalidad estadística va de los salarios reales hacia la productividad laboral.
\end{abstract}

Palabras clave: Venezuela; productividad laboral; salarios; pruebas de causalidad de Granger.

Clasificación JEL: C51; E24; E25; J24; J31; O47.

\section{LABOR PRODUCTIVITY AND REAL WAGE: CAUSAL RELATIONSHIPS IN VENEZUELA}

\begin{abstract}
The discipline of economics has struggled to find a clear or well-established causal relationship between labor productivity and the real wage, despite the close statistical relationship and comovements that several studies report. The policy implications vary drastically depending on what causal link may exist between the two variables. Using conventional Granger causality tests and causality tests in a cointegration environment, with both annual and quarterly data for Venezuela, this article presents evidence showing that statistical causality runs from real wages to labor productivity, a finding which contrasts the orthodox thinking on the subject.
\end{abstract}

Key Words: Venezuela; labor productivity; wages; Granger causality tests.

\footnotetext{
${ }^{a}$ Facultad Latinoamericana de Ciencias Sociales (FLACSO)-Ecuador, Departamento de Desarrollo, Ambiente y Territorio, Ecuador; ${ }^{b}$ Venanalytics, Economic Research Unit, Venezuela. Correos electrónicos: lvvera@flacso.edu.ec y jvera@venanalytics.com, respectivamente.
} 


\section{INTRODUCCIÓN}

Diversos y muy complejos son los factores que a lo largo del tiempo los economistas han encontrado para explicar los cambios en la productividad del trabajo. Smith (1776) se hizo de un éxito indiscutible al destacar el papel estelar que juega la división del trabajo en el sistema de libre empresa. Mientras que Ricardo (1817) y Marx (1867) notaron la influencia de la maquinaria. Rasgos más neoclásicos surgieron con los potenciales efectos que pueden tener los cambios en el precio relativo de los factores de producción sobre la mezcla de capital y trabajo. La inversión en equipos de nueva generación, la adquisición de nuevos conocimientos, la acumulación de destrezas y capital humano, y las tecnologías de la información, son aspectos que también hoy son considerados como factores que inciden decisivamente en el curso que toma la productividad laboral.

Sin embargo, con relación al pago, es decir, al incentivo que representa el salario real, los economistas no encuentran una relación clara o bien establecida con la productividad laboral, a pesar de la estrecha relación que muchos estudios de series estadísticas reportan entre ambas variables. ${ }^{1}$ Basta recordar que la constatación que hace Kaldor (1961) de la constancia de la participación de los salarios en el producto nacional, es un hecho estilizado que supone y contiene implícitamente una relación de largo plazo entre la productividad laboral y el salario real. Lo que se precisa indagar, y es el asunto sobre el cual la literatura teórica y empírica se ha volcado, es si entre estas dos variables hay un vínculo causal.

Existe un enfoque ortodoxo con origen en la teoría neoclásica de la distribución que concibe el vínculo entre estas dos variables como uno, en el que el pago de los factores está gobernado por la productividad. Desde esta perspectiva, el salario real se ajusta a cambios en la productividad marginal del trabajo, y sólo aumentos en la productividad del trabajo justifican incrementos en el salario. De igual manera, el salario real debería ser regulado a la baja en la medida que la productividad marginal del trabajo disminuye. Si en este segundo caso existen impedimentos o rigideces a la baja, el ajuste es por empleo. Por lo demás, en un mundo de mayor o creciente comercio global, mantener los salarios reales a raya y ligados a incrementos en la productividad, es esencial para mantener la competitividad internacional.

1 Esta relación sigue siendo positiva aunque, en los últimos años, estudios para países desarrollados muestran que los índices de productividad laboral aumentaron más que los índices de salario real (OECD, 2015), lo que en efecto constituye uno de los tantos rompecabezas para los analistas. 
Frente a esta perspectiva ortodoxa, otros enfoques alternativos revierten la causalidad que se establece entre la productividad laboral y los salarios. El espacio ha sido fértil para un variado menú de teorías donde los cambios en el salario real bien pueden generar: cambios en el esfuerzo puesto por la fuerza de trabajo, cambios en el costo relativo de los factores que hacen cambiar la mezcla de trabajo y capital, o estímulos a la inversión en capital de nueva cosecha y a cambios tecnológicos (que ulteriormente generan cambios en la productividad), o variaciones en la demanda que impactan sobre el acervo de innovaciones.

En el caso de Venezuela, Baptista (2005) hace una interesante precisión de cuán estrecha ha sido la evolución del salario real y la productividad del trabajo entre 1950 y 2004. Durante ese periodo, la productividad creció a una tasa anual de $1.34 \%$, en tanto que los salarios reales lo hicieron a una tasa de $1.22 \%$. Sin embargo, el autor deja constancia de la trayectoria en U invertida de estas variables a lo largo del tiempo y muestra que a partir de 1977 la tendencia al crecimiento se revierte, por lo que, tanto la productividad como el salario se desploman secularmente. La fase creciente (1950-1977) corresponde con un periodo en el cual el PIB per-cápita de Venezuela se duplica; en tanto que la fase descendente (1977-2004) corresponde a una en que el PIB per-cápita al final del periodo se ubica $25 \%$ por debajo del PIB per cápita inicial. Sin ir al fondo del asunto, Baptista (2005, p. 106) señala que el mercado de trabajo en Venezuela está dominado por "una relación primordial" donde "las variaciones de los salarios reales se ajustan siempre y en todo caso a las variaciones de la productividad". Si tal afirmación tiene asidero empírico será en buena medida objetivo del presente trabajo.

Como se verá a mayor de detalle, son numerosos los estudios empíricos que intentan determinar cuál es el sentido o la relación de causalidad entre el salario real y la productividad del trabajo; sin embargo, la mayor parte de ellos están dirigidos a contrastar esta relación en economías desarrolladas. En este trabajo se presenta evidencia, con datos anuales y trimestrales de ambas series para Venezuela, que muestra que el sentido de la causalidad va de los salarios reales a la productividad laboral.

Incluida la introducción, el estudio se divide en cinco partes: en la segunda sección se presenta un breve resumen del debate teórico. En la tercera sección, la evidencia empírica reportada por los estudios previos encontrados es abordada. En la cuarta sección del trabajo, se usa la tradicional metodología (o prueba) de Granger, así como del contraste de causalidad de Granger en ambiente de cointegración para determinar, con series estadísticas anuales y trimestrales, el sentido de causalidad que los datos muestran para la economía venezolana. El estudio finaliza con breves comentarios finales. 


\section{DEBATE TEÓRICO}

La teoría económica encuentra diversas explicaciones para relacionar la productividad laboral con los salarios reales. Dichas explicaciones pueden en principio dividirse en dos grupos: la teoría ortodoxa o convencional que intenta explicar los cambios de los salarios reales como liderados por cambios en la productividad del trabajo, y ciertas teorías alternativas que buscan más bien revertir la causalidad explicando los cambios de la productividad del trabajo como el resultado de cambios en los salarios reales. A decir verdad, estas teorías no sólo se diferencian en el orden en que establecen la causalidad entre estas dos variables, sino además en los mecanismos que explican esta relación.

La primera explicación de la relación entre estas dos variables proviene de la teoría microeconómica ortodoxa que establece, dados el estado de la tecnología, el precio de los insumos, y el precio de mercado del bien producido, que la relación entre los salarios y la productividad es a través de la curva de la demanda de trabajo. Este enfoque se basa en los primeros estudios de Clark (1899) sintetizados en su conocida obra The distribution of wealth, según la cual "en condiciones de competencia perfecta, los salarios de toda clase de trabajo tienden a igualarse al producto que se pueda atribuir separadamente al trabajo" (Ferguson y Gould, 1980, p. 372). Clark, en efecto, considera que este principio de la productividad marginal determina los salarios. Así, desde una posición de desequilibrio, si la productividad marginal del trabajo es mayor al salario real, las empresas considerarían rentable contratar más mano de obra y pondrían presiones al alza sobre el salario (y a la baja sobre la productividad laboral por los rendimientos decrecientes). En contraste, si la productividad marginal es menor al salario, las empresas encontrarán rentable disminuir la dotación de mano de obra, generando entonces presiones a la baja del salario (y a alza sobre la productividad marginal). El mecanismo genera, de esta manera, una dinámica de estabilidad simple hacia el equilibrio.

El principio donde los salarios están gobernados por la productividad es tan arraigado que no es raro encontrarlo en la literatura que analiza el mercado de trabajo en las economías en desarrollo y menos desarrolladas. Un influyente estudio sobre la productividad en América Latina, publicado por el Banco Interamericano de Desarrollo (Banco Interamericano de Desarrollo [BID], 2010) describe el círculo vicioso de la productividad laboral en la región imponiendo un orden en esta relación en los siguientes términos: 
Como una menor productividad redunda en salarios reales más bajos, es comprensible que los gobiernos traten de mejorar el nivel de vida de los trabajadores con más programas sociales, en particular para los del sector informal. Esto, a su vez, amplía aún más la brecha entre el costo del empleo formal e informal y da lugar a que haya más empleados por cuenta propia y microempresas que no ofrecen cobertura de seguridad social a sus trabajadores. Esta tendencia genera más puestos de trabajo de baja productividad, contrae la oferta de mano de obra para las empresas formales más productivas e impide aumentos de los salarios reales (BID, 2010, p. 14).

En su muy reciente reporte World Economic Outlook para 2017, el Fondo Monetario Internacional (FMI) analiza la lenta dinámica en los niveles salariales a nivel mundial y resume este problema señalando:

En la medida que el crecimiento de la productividad se debilita, manteniendo otros factores constantes, la rentabilidad disminuye junto con la capacidad de las empresas para acomodar aumentos salariales para sus trabajadores existentes, así como su voluntad de atraer nuevos trabajadores con altos salarios. Por lo tanto, el crecimiento salarial tiende debilitarse a medida que el crecimiento de la productividad se ralentiza (Fondo Monetario Internacional [FMI], 2017, p. 75).

Así que, en la perspectiva convencional, el nexo que puede establecerse entre salarios y productividad se ha decantado por una relación donde los salarios están gobernados por la productividad laboral. Entre las implicaciones de política más importantes que se desprenden de esta visión, está la imposibilidad de propiciar aumentos salariales que no afecten negativamente el nivel de empleo, si no están ligados a incrementos previos en la productividad laboral. Por otro lado, si al tema comercial y del posicionamiento en la economía global se remite, mantener los salarios reales a raya ligados a incrementos en la productividad, es esencial para mantener la competitividad internacional.

Pero, desde otra lógica, vale preguntarse si no son los aumentos salariales los que generan una presión sobre las unidades productivas para aumentar la productividad y mantenerse competitivas. Existen, en efecto, enfoques alternativos a la teoría económica ortodoxa que encuentran factible revertir la causalidad que se establece entre los salarios y la productividad laboral. En este terreno, se evidencian un conjunto numeroso de argumentos que justifican y permiten señalar que incrementos (reducciones) en los salarios reales son los que mueven y pueden tener un impacto positivo (negativo) en la productividad del trabajo. 
Storm y Naastepad (2007) apalean al conocido argumento que se desprende de las teorías del "salario eficiencia", mismas que sostienen que la productividad laboral depende positivamente de las remuneraciones salariales al incrementarse el esfuerzo de los trabajadores ante un mayor costo de oportunidad por pérdida de empleo. Lo que ocurre cuando un trabajador que es promovido con un salario más alto, que el que puede ganar en otro puesto de trabajo, se esfuerza aún más para evitar el despido (Gordon, 1994). Otros como Weisskopf et al. (1983) simplemente señalan que mejoras sustanciales en los salarios tendrán como resultado mayor esfuerzo y cooperación por parte de los trabajadores para aumentar su productividad. Sin embargo, esta relación entre salario y esfuerzo en los modelos de salario eficiencia es no-lineal (el esfuerzo marginal es decreciente al salario), lo que acota el impacto que puede ejercer el alza salarial y lleva a las empresas a buscar un salario óptimo que minimiza las horas de trabajo eficiente (que no coincide con el salario que despeja el mercado). Soo Khoon (2009) señala que un efecto económico de tipo salario eficiencia ocurre cuando al aumentar los salarios se reducen las probabilidades de que los trabajadores renuncien, y esto permite a las firmas retener capital humano más experimentado y productivo. Por otra parte, hay quienes enfatizan un efecto no económico, estimando que cuando las empresas y empleados operan bajo restricciones de equidad, un aumento en los salarios reales de los trabajadores tendrá un efecto positivo en la productividad laboral, ya que aumenta el compromiso de los trabajadores con la empresa (Akerlof, 1982). En esta misma dirección, Storm y Naastepad (2007) indican que una firma que establece salarios por debajo de la percepción de salario justo tendrá como resultado un esfuerzo menor por parte de su personal.

Sin embargo, existe otra literatura que presenta la relación entre salario real y productividad del trabajo invocando el efecto sustitución entre mano de obra y bienes de capital. Veerger y Kleinknecht (2011), por ejemplo, señalan que en la consecución de máximos beneficios los incrementos en los costos salariales llevan a las firmas a sustituir trabajo por capital, hasta que la productividad marginal del trabajo se iguala al salario real. Este ajuste se verá reflejado, en definitiva, en un incremento en la productividad marginal del trabajo. Y distintos autores coinciden con esta visión. Por ejemplo, Gordon (1987) y Sylos-Labini (1983) justifican la relación entre salario real y productividad laboral de esta manera.

Sylos-Labini (1983), en particular, llama a este efecto sustitución, "efecto estático" de cambios en el salario sobre la productividad, y argumenta que, dada la tecnología y la organización del proceso productivo, la variación de los salarios con respecto al precio de los bienes de capital genera el cambio en 
la mezcla entre trabajo y bienes de capital que ulteriormente conlleva a cambios en la productividad de los factores. Apela de igual manera a un "efecto dinámico" ligado a la adopción de nuevas tecnologías, en el que si varían los salarios relativos se estimulan cambios tecnológicos y organizacionales; dicha sustitución dinámica implica un ahorro relativo del factor trabajo. En esta misma dirección, Marquetti (2004) parte de la hipótesis de que cambios en los salarios reales generan presión y cierta recompensa a nivel de las firmas para encontrar y adoptar nuevas técnicas con mayor productividad laboral, una posición muy similar a la esgrimida por Marx (1867) en El capital sobre cómo los incrementos en el costo salarial constituyen un incentivo para el progreso tecnológico.

Incrementos en la productividad laboral, inducidos por mejoras salariales, pueden ser concebidos por el efecto de la adopción de capital de "nueva cosecha”. Según Madsen y Damania (2001), si el salario se eleva y genera con ello más inversión en capital fijo, la mera formación de capital de nueva cosecha permite utilizar el servicio del factor trabajo de una forma más productiva y eficiente. En una línea muy similar, Veerger y Kleinknecht (2011) señalan que políticas salariales más agresivas, adoptadas por parte de los sindicatos y empleadores, incentivarán y causarán un remplazo mucho más rápido del capital de vieja cosecha por capital de nueva generación haciendo al factor trabajo mucho más productivo.

Asimismo, desde una perspectiva shumpeteriana, Veerger y Kleinknecht (2011) señalan que en un contexto de mayores salarios reales se acelera el proceso de destrucción creativa, lo que hace que los innovadores tomen mayor dominio de mercado, gracias a la renta monopólica de sus productos y a un tipo de conocimiento del proceso productivo que genera barreras de mercado. La expulsión del mercado de las empresas menos innovadoras, trae como consecuencia una elevación de la productividad media del trabajo a nivel de cada industria.

Cuando cambian los salarios puede existir por igual un efecto de innovación a través de la demanda, según contempla la teoría de empuje de demanda de Schmookler (1966). Al subir los salarios, la demanda efectiva se eleva por la vía del consumo, y una mayor demanda de bienes y servicios supone actividad más innovadora. Por lo tanto, una estrategia de reducción de costos salariales que conlleva a una reducción de la demanda efectiva puede impedir el proceso de innovación y el crecimiento de la productividad laboral. Veerger y Kleinknecht (2014) recuerdan cuán parecida resulta esta conexión entre demanda y productividad desde la tesis de Schmookler, con la llamada Ley Kaldor-Verdoorn. Si en efecto un alza salarial provoca un empuje de demanda 
y de producción, el mecanismo Kaldor-Verdoorn mediaría para promover incrementos de la productividad a través de los rendimientos crecientes.

\section{EVIDENCIA EMPÍRICA}

Pero ¿qué dice la evidencia empírica acumulada sobre la relación causal entre salarios reales y productividad laboral? La mayor parte de los estudios conocidos están dirigidos a contrastar esta relación en economías desarrolladas. Nayaran y Smyth (2009), por ejemplo, lo hacen para las economías del G7 (Canadá, Francia, Alemania, Italia, Japón, Reino Unido y Estados Unidos) apelando al contraste de raíces unitarias y cointegración en un marco de datos de panel y para el periodo 1960-2004. Los autores encuentran que existe un único vector de cointegración donde la productividad es la variable endógena. El estimador de Mínimos Cuadrados Ordinarios Completamente Modificados (FMOLs, por sus siglas en inglés) aplicado al panel indica (con excepción de la economía canadiense) que un incremento de $1 \%$ en el salario real genera un incremento en la productividad laboral entre 0.4 y $1.4 \%$. Veerger y Kleinknecht (2011 y 2014), de igual manera, usan datos de panel controlando por endogeneidad para 19 y 20 países de la Organización para la Cooperación y el Desarrollo Económicos (oECD), respectivamente, para el periodo 19602004. En ambos ejercicios los autores encuentran que cambios en $1 \%$ en la tasa de crecimiento del salario real inducen cambios en el rango de 0.35-0.46 puntos porcentuales en el crecimiento de la productividad del trabajo. Storm y Naastepad (2009) realizan el ejercicio para un grupo de 20 países de la OECD, durante el periodo 1984-2004, y encuentran que el impacto es de aproximadamente $0.3 \%$. Por su parte, Madsen y Damania (2001) usan un pool de datos de sección transversal y series de tiempo para la industria manufacturera de 22 países de OECD y estiman un impacto positivo de los salarios reales sobre la productividad del trabajo y del capital. Hein y Tarassow (2009) emplean series temporales anuales (1960-2007) para seis países de la OECD (Alemania, Francia, Holanda, Austria, Reino Unido y Estados Unidos) y encuentran en sus regresiones, usando otras variables de control, un impacto positivo de cambios en el salario real sobre la productividad. El impacto (dependiendo de la economía) se mueve en un rango entre 0.54 y $0.23 \%$.

Entre los estudios para países individuales destaca el trabajo de Weisskopf et al. (1983) quienes usan datos anuales del sector privado no agrícola de Estados Unidos, para el periodo 1948-1979, y encuentran, en regresiones de series de tiempo, que los cambios en la productividad laboral están influenciados, 
entre otras variables, por el salario. Simultáneamente, Sylos-Labini (1983) presenta un ejercicio para Estados Unidos (1952-1981) y la economía italiana (1962-1980) donde usan datos de series de tiempo para la economía como un todo y para el sector industrial en particular, corrobora así la hipótesis de la incidencia del costo de laboral sobre la productividad del trabajo. Lucidi (2008) usa micro-datos a nivel de firmas provenientes del Instituto Italiano de Entrenamiento Vocacional e intenta replicar la especificación de Sylos-Labini (1983), encontrando para el sector empresarial italiano una elasticidad de la productividad del trabajo al costo laboral positiva y menor que la unidad. Mientras que Valadkhani (2003) reconoce, en datos de serie de tiempo anual para Australia (1970-2001), un efecto positivo del crecimiento del salario sobre el crecimiento de la productividad, con una elasticidad de corto plazo en un modelo de corrección de errores de 0.22. Upender y Sujan (2008) usan datos agregados para el sector industrial en India y encuentran, para el periodo 1980-2005, un vector de cointegración para el salario real promedio y la productividad media del trabajo. Un modelo de corrección de errores revela que en el corto plazo la elasticidad de la productividad a cambios en el salario real es de 0.73 . Yusof (2008) está entre los pocos estudios que usa datos trimestrales (1992:1-2005:3), en este caso para la economía de Malasia. El estudio encuentra una relación de largo plazo entre la productividad laboral, el empleo y el salario real. Sin embargo, los resultados a partir de un Modelo de Vectores de Corrección de Errores (VECM) muestran que el salario real no es una variable débilmente exógena y que, por el contrario, se ajusta para mantener la relación de cointegración en el modelo.

Existe también un buen número de ejercicios empíricos que se enfocan en contrastar la causalidad estadística entre la productividad laboral y el salario real. Por ejemplo, Marquetti (2004) investiga si existe una relación de largo plazo para salarios reales y productividad del trabajo en la economía norteamericana usando datos que van de 1869 a 1999. Las pruebas de cointegración indican la existencia de una relación de largo plazo entre estas variables, mientras las pruebas de causalidad de Granger en un ambiente de cointegración señalan que los salarios reales causan la productividad laboral. Strauss y Wohar (2004) usan datos de panel para 459 sectores de la industria manufacturera norteamericana para el periodo 1956-1996. En sus hallazgos observan, al aplicar la misma prueba de Granger en ambiente de cointegración, una relación de causalidad bidireccional entre salarios reales y productividad laboral. Un estudio de series de tiempo anuales para Tanzania para el periodo 1967-2010 es conducido por Islam et al. (2015). Las pruebas de causalidad bi-variada de Granger indican que la productividad laboral causa estadísticamente mo- 
vimientos del salario real. Por su parte, apelando a datos de serie de tiempo anual para Australia para el periodo 1965-2007, Kumar et al. (2012) investigan la causalidad entre la productividad laboral, la tasa de inflación y el salario real, usando estimadores VECM, FMOLS y Autorregresivos de Retardos Distribuidos (ARDL). Consistentemente encuentran que el impacto de cambio en los salarios reales sobre la productividad del trabajo es positivo y con una elasticidad que se ubica entre 0.5 y 0.8. En la misma línea, Yildrim (2015) aplica una metodología similar (causalidad en ambiente de cointegración), pero con datos trimestrales y para la industria manufacturera en Turquía, y para el periodo 1988:1-2012:2. Las pruebas de causalidad indican que en efecto los salarios reales causan la productividad laboral, pero que la productividad no causa los salarios. Méndez y Hernández (2014) usan la misma metodología para datos de panel del sector manufacturero de la economía de Colombia y para 22 entidades estadales, aunque no encuentra una relación de largo plazo entre el salario y la productividad. En consecuencia, los autores usan un modelo VAR en diferencias. Las pruebas de causalidad en ambiente VAR muestran que la relación es bi-direccional. Karaalp-Orhan (2017) investiga la relación entre productividad laboral, salarios reales promedio y tasa de desempleo también para Turquía, empleando el procedimiento de pruebas límites (bounds testing) al interior de un modelo ARDL y aplicando la prueba de causalidad Toda-Yamamoto con datos trimestrales para el periodo 2007:01-2016:04. Sus resultados encuentran que aun cuando las variables están cointegradas y existe un impacto de largo plazo del salario real sobre la productividad, en el corto plazo no existe ningún tipo de causalidad estadística entre estas variables. Pyo (2018) utiliza datos anuales para las economías de Japón, China y Corea de Sur (1970-2014) y encuentra una relación de cointegración entre la productividad laboral y el salario real en las tres economías. Las pruebas de causalidad bi-variada de Granger indican que en Corea del Sur y China los salarios causan la productividad del trabajo, no así en el caso de Japón. De cualquier forma, la causalidad en reverso (de la productividad al salario) no parece existir en ninguna de estas economías. Brida et al. (2010) usan datos para la industria del turismo en México entre 1970 y 2004. Los autores encuentran una relación de largo plazo entre el salario real y la productividad laboral y, en las pruebas de causalidad de Granger y con funciones de respuesta al impulso, corroboran que la causalidad va de los salarios reales hasta la productividad.

En consecuencia, no son pocos los estudios empíricos que corroboran que es el salario real la variable que empuja los cambios en la productividad y no viceversa. 


\section{PRUEBAS EMPÍRICAS PARA EL CASO DE LA ECONOMÍA VENEZOLANA: DATOS, METODOLOGÍA Y RESULTADOS}

Para despejar el asunto sobre la relación que se da entre el salario real y la productividad del trabajo, se usaron datos para la economía venezolana y dos pruebas de causalidad estadística: la prueba bi-variada de Granger (1969) y Sims (1972), así como la prueba de causalidad de corto plazo en ambiente de cointegración sugerida por Marquetti (2004) y Hossain (2005). Además de series temporales con datos anuales para el periodo 1967-2013, pero también series de datos trimestrales para el periodo 1998:1-2013:4.

\section{Relación para datos anuales}

Para el análisis con datos anuales se usarán, por el momento, dos series estadísticas:

a) Un índice del comportamiento del salario real (ISR) en Venezuela, serie que es tomada de las estadísticas del Banco Central de Venezuela (BCV) para los años 1967-1998 y cuyo año base es 1984. A partir de 1998 y hasta 2013, se empalma con la variación de la serie anualizada del índice de remuneraciones real del mismo BCV.

b) Un indicador de la productividad media del trabajo (PL) que se calcula como un ratio entre el PIB anual en términos reales publicado por el BCV y la población ocupada reportada por el Instituto Nacional de Estadística (INE).

El comportamiento en el tiempo de estas dos variables tomadas en logaritmo, para el periodo 1968-2013, puede observarse a partir de las figuras 1 y 2. El recorrido de ambas series es similar al de los datos reportados por Baptista (2005), y parece claro que después de un periodo de ascenso ambas variables llegan a un pico a mediados de los ańos setenta y decrecen tendencialmente hasta principios de la década de 2000, momento a partir del cual la productividad muestra una leve recuperación, que no muestra a decir verdad el salario real. No obstante, conviene precisar la muy alta asociación positiva entre el salario real y la productividad laboral en series anuales, con un índice de correlación de 0.8719 . 
Leonardo Vera y Juan Andrés Vera

Figura 1. Productividad laboral anual (1967-2013)

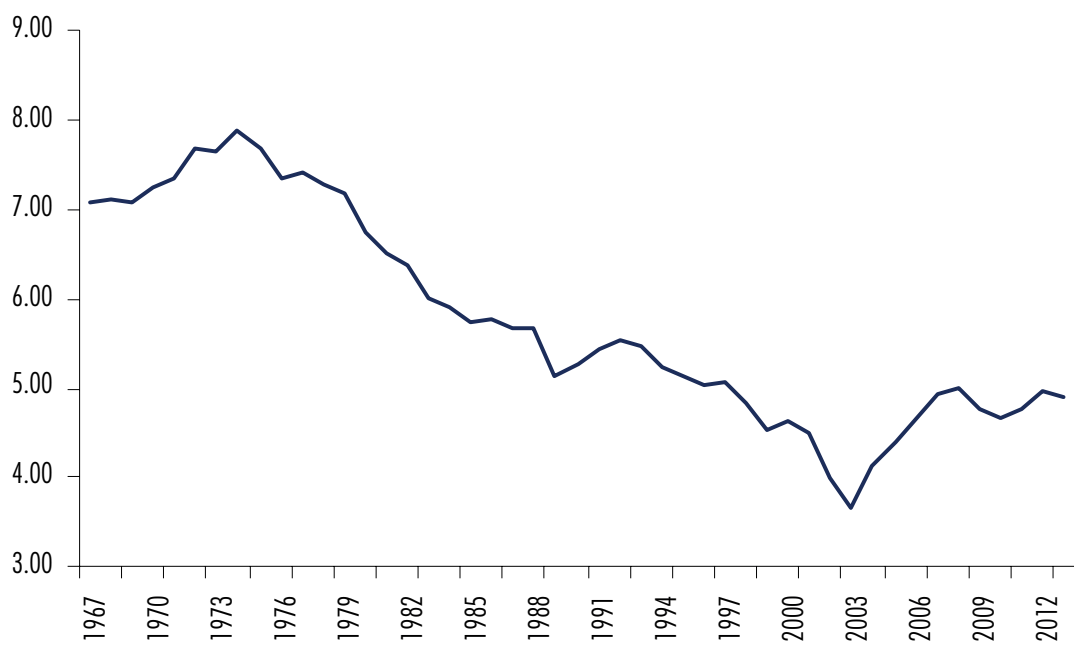

Fuente: elaboración propia.

Figura 2. Índice de salario real anual (1967-2013)

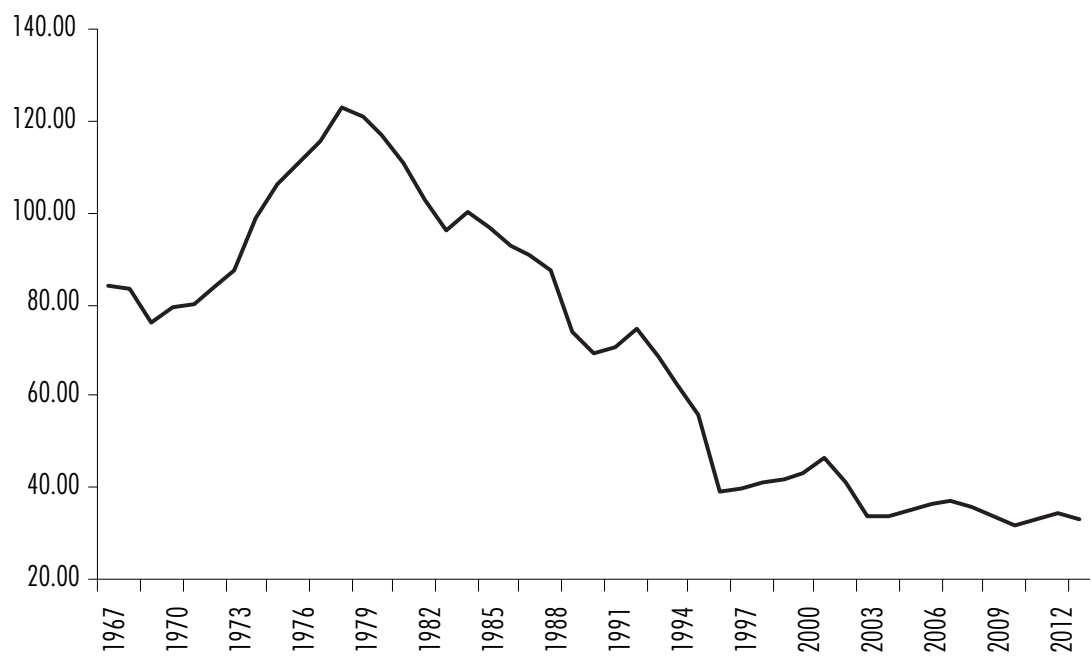

Fuente: elaboración propia. 
A los efectos de hacer un primer contraste de causalidad estadística se usará la prueba de Granger (1969) y Sims (1972). Sin embargo, para la realización de esta prueba de causalidad es necesario que las variables sean estacionarias para así evitar resultados espurios en regresiones con series de tiempo no estacionarias. En consecuencia, previo se analizarán la estacionariedad de las series aplicando las pruebas más convencionales de Dickey-Fuller Aumentada (DFA) y de Phillips-Perron (PP), pero también la prueba raíz unitaria de punto de corte (Breakpoint Unit Root, BPUR) que toma en cuenta la posible presencia de cambios estructurales en las series. El contraste de hipótesis para estas pruebas es el mismo, tanto para la hipótesis nula como para la hipótesis alternativa. En esencia, se contrasta la hipótesis nula de un proceso con raíz unitaria $v$ s. la hipótesis alterna que señala que el proceso generador de los datos es un proceso estacionario.

La tabla 1 recoge los resultados de aplicar las dos pruebas de raíces unitarias sobre la serie de salario real y PL. Para ambas variables en nivel los valores calculados por las pruebas DFA, PP (sin intercepto y sin tendencia) son mayores a los valores críticos, y las probabilidades son todas mayores a 0.05 , por lo que se falla en rechazar la hipótesis nula de que hay presencia de una raíz unitaria. La prueba BPUR de igual manera rechaza la hipótesis nula de presencia de una raíz unitaria de las series en nivel. En consecuencia, las variables PL e ISR no son estacionarias en nivel. Al tomar las variables en primera diferencia, los valores calculados de las pruebas son menores a los valores críticos, y las probabilidades son todas menores a 0.05 por lo que se rechaza entonces la hipótesis

Tabla 1. Pruebas de raíces unitarias (datos anuales)

\begin{tabular}{lcccccccccc}
\hline Variables & $\begin{array}{c}\text { Prueba } \\
\text { Dickey-Fuller } \\
\text { Aumentada }\end{array}$ & $\begin{array}{c}\text { Valores } \\
\text { críticos } \\
\text { prueba } \\
\text { DFA }\end{array}$ & Prob. & $\begin{array}{c}\text { Prueba } \\
\text { Phillips- } \\
\text { Perron }\end{array}$ & $\begin{array}{c}\text { Valores } \\
\text { críticos } \\
\text { P.P }\end{array}$ & $\begin{array}{c}\text { Prob. } \\
\text { Prueba } \\
\text { Breakpoint } \\
\text { Unit Root }\end{array}$ & $\begin{array}{c}\text { Valores } \\
\text { críticos } \\
\text { BPUR }\end{array}$ & Prob. & Resultado \\
\hline LnPL & -1.3228 & -1.9481 & 0.1694 & -1.1903 & -1.9481 & 0.2106 & -2.741 & -4.4436 & 0.8141 & $p=1$ \\
LnISR & -1.6159 & -1.9481 & 0.0993 & 1.4286 & -1.9481 & 0.1408 & -2.745 & -4.4436 & 0.8124 & $p=1$ \\
DLnISL & -4.5983 & -1.9483 & 0.0000 & -4.5983 & -1.9483 & 0.0000 & -5.9495 & -4.4436 & 0.0100 & $p<1$ \\
DLnPL & -4.9613 & -1.9483 & 0.0000 & -4.8830 & -1.9483 & 0.0000 & -6.0521 & -4.4436 & 0.0100 & $p<1$ \\
\hline
\end{tabular}

Notas: PL = Productividad Laboral; ISR = Índice de Salario Real; DFA and PP sin rezago y sin tendencia ni intercepto. Breakpoint Unit Root sin rezago y con intercepto.

Fuente: elaboración propia. 
nula de que las variables son no-estacionarias. En conclusión, ambas pruebas indican que la PL y el ISR son variables estacionarias en primera diferencia.

En conocimiento de que ambas variables son I(1), se procederá a aplicar la prueba de causalidad de Granger, que consiste en correr dos regresiones dinámicas sobre las variables $X_{t}$ y $Y_{t}$, a decir:

Una regresión no restringida

$$
Y=\sum_{i=1}^{m} \alpha_{i} Y_{t-i}+\sum_{i=1}^{m} \beta_{i} X_{t-i}+e_{t}
$$

Una regresión restringida

$$
Y=\sum_{i=1}^{m} \alpha_{i} Y_{t-i}+e_{t}
$$

La suma de los residuos al cuadrado de cada regresión se usa para calcular un estadístico $\mathrm{F}$ y así probar si los coeficientes son significativamente distintos de cero. Si no lo son, se rechaza la hipótesis que señala de " $X$ no causa a $Y$ ". Cambiando las variables $(X$ por $Y$ ) se corren regresiones similares para probar la hipótesis que dice que " $Y$ no causa a $X$ ". Nótese que el número de rezagos es arbitrario, por lo que la prueba suele correrse con distintos niveles de rezago. La prueba de causalidad de Granger se aplica entonces para determinar qué variable tiene incidencia o causa efectos sobre la otra variable y se logra contrastando las hipótesis nulas que establecen que la variable " $X$ no causa a la variable $Y$ " y que la variable " $Y$ no causa a la variable $X$ ".

En la tabla 2 se muestran los resultados de aplicar la prueba de causalidad de Granger para datos anuales. El ejercicio se realizó en niveles y en diferencias, con dos y tres rezagos. La estructura de rezagos obedece el criterio de elegir sólo aquellos casos donde los errores son esféricos (normales, homocedásticos y no correlacionados) en una estructura VAR en niveles conteniendo ambas variables. Las probabilidades arrojadas por la prueba de causalidad en niveles utilizando dos rezagos son iguales a 0.009 y de 0.079 ; mientras que utilizando tres rezagos arrojaron valores iguales a 0.003 y 0.53 . Lo anterior indicaría que, en niveles, la variable ISR causa estadísticamente o mueve a la PL; más se falla en rechazar la hipótesis nula que dice que la productividad del trabajo no causa al ISR. Sin embargo, como señalan Green (1997) y Wooldridge (2013), si las variables incluidas en las regresiones (1) y (2) no son 
Tabla 2. Pruebas de causalidad Granger con datos anuales (1967-2013)

\begin{tabular}{lccc}
\hline Hipótesis nula & Número de rezagos & Estadístico F & Probabilidad \\
\hline LnISR no causa Granger LnPL & 2 & 5.24 & 0.009 \\
LnPL no causa Granger LnISR & 2 & 2.64 & 0.079 \\
LnISR no causa Granger LnPL & 3 & 5.29 & 0.003 \\
LnPL no causa Granger LnISR & 3 & 2.81 & 0.053 \\
DLnISR no causa Granger DLnPL & 2 & 7.77 & 0.001 \\
DLnPL no causa Granger DLnISR & 2 & 0.39 & 0.679 \\
DLnISR no causa Granger DLnPL & 3 & 4.74 & 0.007 \\
DLnPL no causa Granger DLnISR & 3 & 0.42 & 0.736 \\
\hline
\end{tabular}

Notas: PL = Productividad Laboral; ISR = Índice de Salario Real; DFA and PP sin rezago y sin tendencia ni intercepto; Breakpoint Unit Root sin rezagos y con intercepto.

Fuente: elaboración propia.

estacionarias, los coeficientes estarán sesgados y la inferencia estadística sería un ejercicio de dudosa validez.

En consecuencia, es preferible tomar como válida la prueba de Granger incorporando las variables en diferencias en las regresiones. Se observa que, con dos y tres rezagos anuales, las probabilidades arrojadas con las variables en diferencia indican esencialmente lo mismo. Se rechaza la hipótesis nula de que el ISR no causa o no tiene efectos sobre la PL y se falla en rechazar la hipótesis nula que dice que la productividad laboral no causa al IsR.

Para observar qué tan robusto es este resultado, se puede realizar un contraste de causalidad de Granger en ambiente de cointegración. A los efectos se precisa previamente constatar que las variables presentan una relación de equilibrio de largo plazo en forma bi-variada.

Si las variables son I(1), como es el caso en estudio, es posible que estén cointegradas y que exista entre ellas una relación de largo plazo. De esa manera, la teoría de la cointegración establece que aún en presencia de variables no estacionarias en nivel, puede existir una combinación lineal entre éstas de tipo estacionario. Para determinar si las variables salario real y productividad laboral están cointegradas, y dado que se está en presencia de una relación bivariada, se realiza la prueba de Engle y Granger (1987) que intenta contrastar 
de la hipótesis de si los residuales de la regresión son estacionarios. ${ }^{2}$ El contraste para determinar si los residuales de la regresión son o no estacionarios supone aplicar la prueba de DFA a los residuales estimados.

La tabla 3 muestra el valor de los estimadores (coeficientes) de las regresiones de la PL como variable dependiente y del ISR como variable explicativa. También se muestra la prueba DFA aplicada a los residuos de las regresiones y las probabilidades de cada contraste para distintos números de rezagos. Allí se observa que la prueba DFA sobre los residuos de la regresión con un rezago arroja una probabilidad de 0.058 ; es decir, al $10 \%$ se rechaza la hipótesis nula de que los residuos son no estacionarios, mientras que las pruebas sobre los residuos de la regresión con dos, tres y cuatro rezagos arrojan probabilidades de $0.045,0.026$ y 0.018 , respectivamente, y son significativos al $5 \%$, lo que quiere decir que son estacionarios. De esta forma, se puede afirmar que sí existe una relación de largo plazo entre la PL y el ISR para datos anuales. ${ }^{3}$

Tabla 3. Pruebas de cointregración sobre los residuos (datos anuales)

\begin{tabular}{llccccc}
\hline Variable & $\begin{array}{l}\text { Variable } \\
\text { regresora }\end{array}$ & Coeficiente & Beta & $\begin{array}{c}\text { Prueba Dickey- } \\
\text { Fuller Aumentada }\end{array}$ & $\begin{array}{c}\text { Número de } \\
\text { rezagos }\end{array}$ & Probabilidad \\
\hline LnPL & LnISR & 13.959 & 0.384 & -1.876 & 1 & 0.058 \\
LnPL & LnISR & 13.959 & 0.384 & -1.993 & 2 & 0.045 \\
LnPL & LnISR & 13.959 & 0.384 & -2.232 & 3 & 0.026 \\
LnPL & LnISR & 13.959 & 0.384 & -2.389 & 4 & 0.018 \\
\hline
\end{tabular}

Fuente: elaboración propia.

Si existe una relación de largo plazo entre las dos variables involucradas en el análisis, entonces una relación de causalidad también existe. Sin embargo, las pruebas de cointegración no proveen información alguna sobre la dirección de la causalidad. Es aquí donde las pruebas de causalidad de Granger en ambiente de cointegración pueden ser útiles. En presencia de cointegración las pruebas bi-variadas dependen de los siguientes modelos de corrección de errores:

2 Hatanaka (1996) señala que este procedimiento es apropiado cuando se trata de dos variables y un posible vector de cointegración.

3 El signo y el valor del coeficiente "beta" (0.38) indica una relación positiva en el largo plazo entre las variables, pero otros factores pueden estar afectando el comportamiento de la productividad laboral. 


$$
\begin{aligned}
& \Delta L n P L=c_{1}+\rho_{1} E C_{t-1}+\sum_{i=1}^{p} \alpha_{i} \Delta L n I S R_{t-i}+\sum_{i=1}^{p} \beta_{i} \Delta L n P L_{t-1}+U_{t} \\
& \Delta L n I S R=c_{2}+\rho_{2} E C_{t-1}+\sum_{i=1}^{p} \gamma_{i} \Delta L n P L_{t-i}+\sum_{i=1}^{p} \theta_{i} \Delta L n I S R_{t-1}+U_{t}
\end{aligned}
$$

Las ecuaciones (3) y (4) indican que los cambios en la variable dependiente $Y_{t}$ dependen no sólo de los cambios en $X_{t}$, sino también del desequilibrio pasado $(Y-k X)_{t-1}$ representado por el llamado término de corrección de error. ${ }^{4}$ Siguiendo a Hossain (2005), dos tipos de pruebas de causalidad de Granger, de corto y largo plazo, pueden hacerse en ambiente de cointegración. Es de particular interés la causalidad de corto plazo. Una prueba $F$ de significación conjunta de los estimadores $\Sigma \alpha_{i}, \Sigma \gamma_{i}$, para $i=1,2 \ldots$, en cada una de las especificaciones anteriores, permite determinar si existe una relación de causalidad o no de corto plazo entre las variables involucradas. Causalidad desde la variable del lado derecho hacia la variable dependiente existirá, si los estimadores antes señalados de la regresión en forma conjunta son distintos de cero. Los resultados para estructuras desde uno hasta cuatro rezagos son presentados en la tabla $4 .^{5}$

Las pruebas en regresiones hasta con tres rezagos indican, en un primer caso, que los estimadores no son distintos de cero y que el ISR en primera diferencia causa el crecimiento de la productividad laboral. Sólo en una estructura dinámica de cuatro rezagos, el crecimiento del salario no causa al crecimiento de la productividad laboral (al 5\% de significación). En contraste, en un segundo caso como se muestra en el modelo de corrección de errores 2 , la evidencia no apunta a una causalidad desde la productividad hasta los salarios, pues en ninguna estructura de rezagos la hipótesis nula de significación conjunta de los parámetros $\gamma_{i}$ puede ser rechazada.

Así que con datos anuales se obtienen resultados robustos que indican que estadísticamente y, sobre todo, en el caso de la economía venezolana si existe una relación causal irá del ISR hasta la productividad laboral.

4 Por tratarse de pruebas de causalidad, las especificaciones (3) y (4) no incluyen como regresores las variables independientes sin rezagos.

5 Una estructura hasta con cuatro rezagos garantiza que los errores en cada estimación son normales, homocedásticos y sin correlación serial. 
Tabla 4. Modelo de corrección de errores con datos anuales

\begin{tabular}{|c|c|c|}
\hline \multicolumn{3}{|c|}{ Modelo de corrección de errores 1 con datos anuales } \\
\hline \multicolumn{3}{|c|}{$\Delta L n P L=c_{1}+\rho_{1} E C_{t-1}+\sum_{i=1}^{p} \alpha_{i} \Delta L n I S R_{t-i}+\sum_{i=1}^{p} \beta_{i} \Delta L n P L_{t-1}+U_{t}$} \\
\hline Número de rezagos & Estadistico $F$ & Probabilidad \\
\hline 1 & 5.3359 & 0.0087 \\
\hline 2 & 3.4604 & 0.0253 \\
\hline 3 & 2.9254 & 0.0337 \\
\hline 4 & 2.2592 & 0.0699 \\
\hline \multicolumn{3}{|c|}{ Modelo de corrección de errores 2 con datos anuales } \\
\hline \multicolumn{3}{|c|}{$\Delta L n I S R=c_{2}+\rho_{2} E C_{t-1}+\sum_{i=1}^{p} \gamma_{i} \Delta L n P L_{t-i}+\sum_{i=1}^{p} \theta_{i} \Delta L n I S R_{t-1}+U_{t}$} \\
\hline Número de rezagos & Estadistico $F$ & Probabilidad \\
\hline 1 & 0.9677 & 0.3884 \\
\hline 2 & 1.2623 & 0.3006 \\
\hline 3 & 0.9424 & 0.4504 \\
\hline 4 & 1.0243 & 0.4184 \\
\hline
\end{tabular}

Fuente: elaboración propia.

\section{Relación con datos trimestrales}

Los datos trimestrales para las variables en estudio se toman para el periodo más largo conseguido que va desde 1998:1 a 20013:4. Corresponde a 64 observaciones para cada serie. Las series han sido construidas de la siguiente manera:

a) El ISR ha sido calculado como la razón entre el índice de remuneraciones total que reporta trimestralmente el BCV y el Índice de Precios al Consumidor con año base 2007.

b) La PL se ha calculado dividiendo el PIB trimestral reportado por el BCV entre la población ocupada cada trimestre reportada por el INE. 
Como puede observarse a partir de las figuras 3 y 4 y para datos trimestrales, tanto la PL como el índice de salario (para el periodo 1998-2013), no muestran asociación a lo largo del tiempo. De hecho, el índice de correlación arroja un valor de -0.0420 . La productividad laboral muestra una tendencia decreciente desde sus inicios y hasta 2002 cuando sufre una estrepitosa caída; de este año en adelante la tendencia de la variable es creciente para estabilizarse hacia el final de la serie. En lo que toca al ISR, éste tiende a crecer desde sus inicios y hasta mediados de 2001, cuando sufre una fuerte caída. A partir de 2004, el salario real exhibe una leve recuperación hasta 2008, cuando vuelve a decrecer y ganar nuevo impulso a partir de 2010.

Antes de llevar a cabo la prueba de causalidad de Granger se requiere realizar pruebas de raíces unitarias. En la tabla 5 se muestra a las variables PL e ISR en nivel y en primera diferencia. Las probabilidades arrojadas por las pruebas de DFA, PP у BPUR indican que las variables productividad media del trabajo e ISR son estacionarias en primera diferencia.

Figura 3. Productividad laboral trimestral (1098-4Q13)

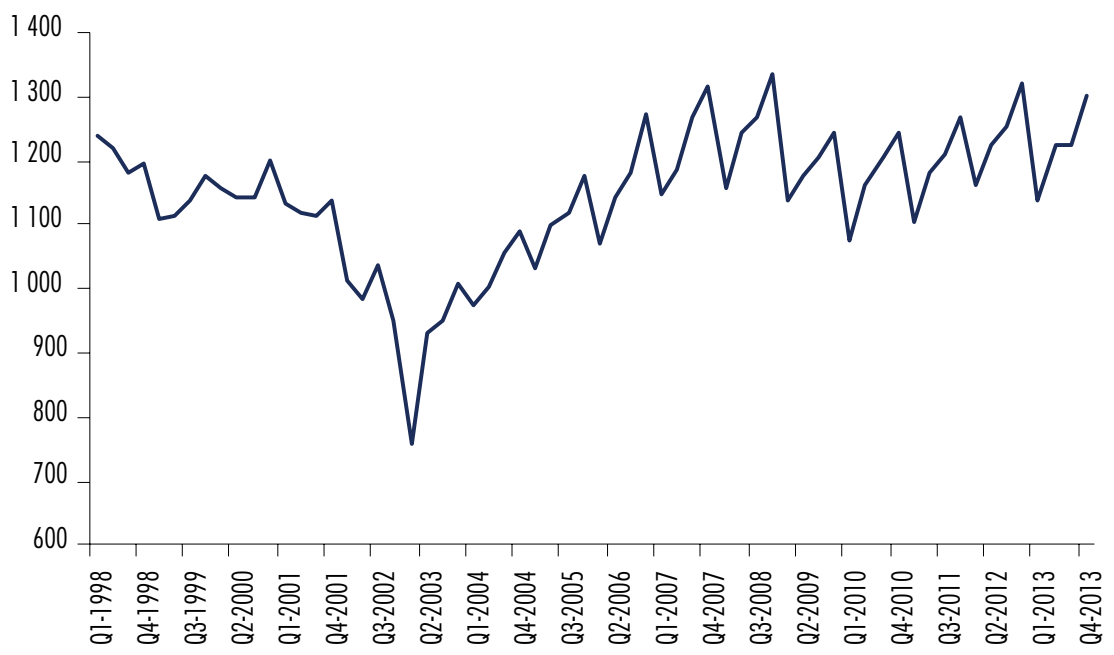

Fuente: elaboración propia. 
Figura 4. Índice de salario real trimestral (1Q98-4Q13)

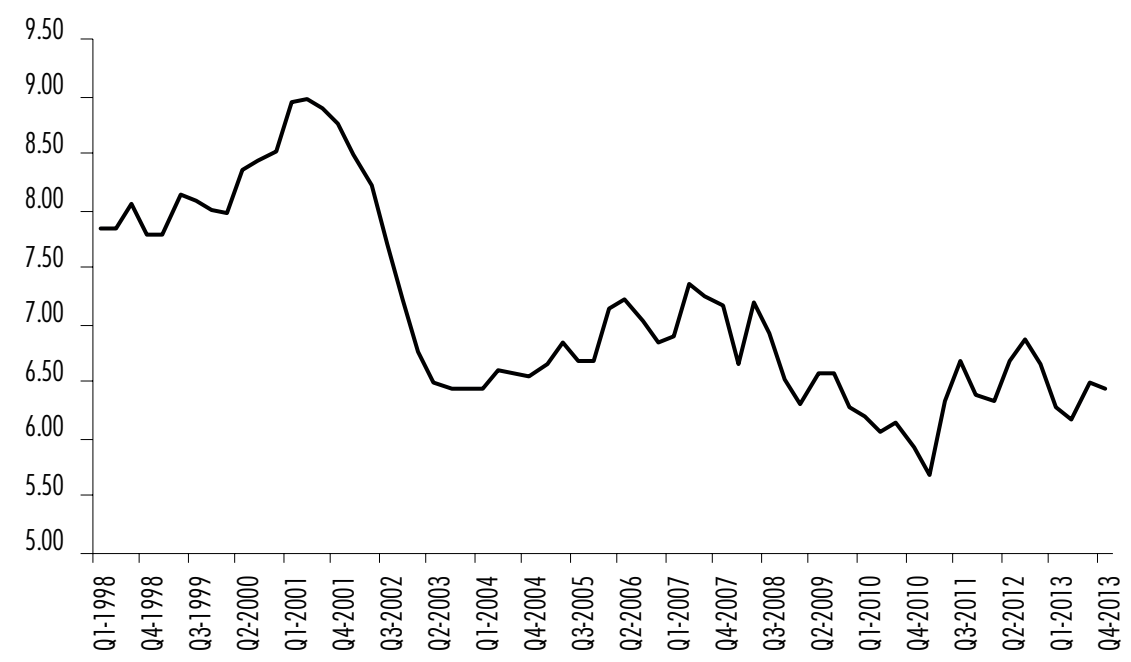

Fuente: elaboración propia.

Tabla 5. Pruebas de raíces unitarias (datos trimestrales)

\begin{tabular}{lcccccccccc}
\hline Variables & $\begin{array}{c}\text { Prueba } \\
\text { Dickey- } \\
\text { Fuller } \\
\text { Aumentada }\end{array}$ & $\begin{array}{c}\text { Valores } \\
\text { críticos } \\
\text { prueba } \\
\text { DFA }\end{array}$ & Prob. & $\begin{array}{c}\text { Prueba } \\
\text { Phillips- } \\
\text { Perron }\end{array}$ & $\begin{array}{c}\text { Valores } \\
\text { críticos } \\
\text { P.P }\end{array}$ & Prob. & $\begin{array}{c}\text { Prueba } \\
\text { Breakpoint } \\
\text { Unit Root }\end{array}$ & $\begin{array}{c}\text { Valores } \\
\text { críticos } \\
\text { BPUR }\end{array}$ & Prob. & Resultado \\
\hline LnPL & 0.2805 & -1.9464 & 0.7639 & 0.1410 & -1.946 & 0.7235 & -3.3183 & -4.4436 & 0.4932 & $p=1$ \\
LnISR & -0.7264 & -1.9460 & 0.3980 & -0.7297 & -1.946 & 0.3965 & -4.2664 & -4.4436 & 0.0809 & $p=1$ \\
DLnISL & -6.8157 & -1.9461 & 0.0000 & -6.8318 & -1.9461 & 0.0000 & -6.9767 & -4.4436 & 0.0100 & $p<1$ \\
DLnPL & -3.3044 & -1.9464 & 0.0013 & -17.5496 & -1.9461 & 0.0000 & -6.8036 & -4.4436 & 0.0100 & $p<1$ \\
\hline
\end{tabular}

Notas: PL = Productividad Laboral; ISR = Índice de Salario Real; DFA and PP sin rezago y sin tendencia ni intercepto. Breakpoint Unit Root sin rezago y con intercepto.

Fuente: elaboración propia.

El siguiente paso consiste en hacer las pruebas de causalidad. En la tabla 6 se puede observar la causalidad de Granger de las variables ISR y productividad media del trabajo, pero para datos trimestrales. La prueba se presenta con las variables en niveles y en diferencias con dos y tres rezagos, por ser las estructuras de rezagos que garantizan errores esféricos. 
Tabla 6. Pruebas de causalidad Granger para ISR y PL (datos trimestrales)

\begin{tabular}{lccc}
\hline Hipótesis nula & Número de rezagos & Estadístico F & Probabilidad \\
\hline LnISR no causa Granger LnPL & 2 & 8.52 & 0.000 \\
LnPL no causa Granger LnISR & 2 & 2.14 & 0.126 \\
LnISR no causa Granger LnPL & 3 & 9.57 & 0.000 \\
LnPL no causa Granger LnISR & 3 & 1.13 & 0.345 \\
DLnISR no causa Granger DLnPL & 2 & 5.55 & 0.006 \\
DLnPL no causa Granger DLnISR & 2 & 1.09 & 0.342 \\
DLnISR no causa Granger DLnPL & 3 & 5.03 & 0.003 \\
DLnPL no causa Granger DLnISR & 3 & 1.90 & 0.139 \\
\hline
\end{tabular}

Fuente: elaboración propia.

Si la atención es puesta en las probabilidades arrojadas por la prueba con datos en diferencia y utilizando dos y tres rezagos, los resultados son 0.006 y 0342 ; y 0.003 y de 0.139 , respectivamente. Por lo tanto, se rechaza la hipótesis nula de que el ISR no causa o no tiene efectos sobre la productividad media del trabajo, pero no es posible rechazar la hipótesis que seńala que la dinámica de la productividad del trabajo no causa la dinámica salarial en términos reales.

De igual manera que con los datos anuales, se procederá a realizar las pruebas de causalidad en ambiente de cointegración, pero para datos trimestrales. Para determinar si las variables en términos trimestrales están cointegradas y ya que se está ante la presencia de una relación bi-variada, se realiza la prueba de Engle y Granger cuyos resultados se muestran en la tabla 7.

La tabla 7 muestra las regresiones de la productividad media del trabajo usando como variable explicativa el ISR, así como los resultados de las pruebas de estacionariedad (con distintos números de rezagos) aplicada a los residuos de la regresión, con el objeto de conocer si son estacionarios y consistentes. Se puede observar que la prueba DFA sobre los residuos de la regresión indica que los residuos son estacionarios al 5\% de significación para regresiones con uno y cuatro rezagos. Para dos rezagos los resultados son significativos al 10\%. En general, las pruebas tienden a confirmar que los residuos de la regresión 
son estacionarios y por ello se puede concluir, a través del método de Engle y Granger, que las variables están cointegradas y forman una relación de largo plazo.

Las pruebas de causalidad en ambiente de cointegración son reportadas en la tabla 8. Se puede observar que independiente del número de rezagos, el ISR causa la productividad del trabajo. No obstante, la no causalidad desde la productividad laboral hasta el ISR no puede ser rechazada, al menos en los rezagos de orden dos, tres y cuatro. Si bien esto no contraviene los resultados antes encontrados, introduce la posibilidad de que en datos trimestrales la causalidad sea bi-direccional.

Tabla 7. Pruebas de cointregración sobre los residuos (datos trimestrales)

\begin{tabular}{llccccc}
\hline Variable & $\begin{array}{l}\text { Variable } \\
\text { regresora }\end{array}$ & Coeficiente & Beta & $\begin{array}{c}\text { Prueba Dickey- } \\
\text { Fuller Aumentada }\end{array}$ & $\begin{array}{c}\text { Número de } \\
\text { rezagos }\end{array}$ & Probabilidad \\
\hline LnPL & LnISR & 14.182 & -0.035 & -2.201 & 1 & 0.027 \\
LnPL & LnISR & 14.182 & -0.035 & -1.695 & 2 & 0.085 \\
LnPL & LnISR & 14.182 & -0.035 & -1.076 & 3 & 0.252 \\
LnPL & LnISR & 14.182 & -0.035 & -1.960 & 4 & 0.048 \\
\hline
\end{tabular}

Fuente: elaboración propia.

Tabla 8. Modelo de corrección de errores con datos trimestrales

\begin{tabular}{lcc}
\hline \multicolumn{3}{c}{ Modelo de corrección de errores 7 con datos trimestrales } \\
\hline \multicolumn{2}{c}{$\Delta L n P L=c_{1}+\rho_{1} E C_{t-1}+\sum_{i=1}^{p} \alpha_{i} \Delta L n I S R_{t-i}+\sum_{i=1}^{p} \beta_{i} \Delta L n P L_{t-1}+U_{t}$} \\
\hline Número de rezagos & Estadístico F & Probabilidad \\
\hline 1 & 7.1241 & 0.0017 \\
2 & 5.4194 & 0.0024 \\
3 & 6.0247 & 0.0004 \\
4 & 5.0527 & 0.0008 \\
\hline
\end{tabular}


Tabla 8. Modelo de corrección de errores con datos trimestrales (continuación)

\begin{tabular}{lcc}
\hline \multicolumn{3}{c}{ Modelo de corrección de errores 2 con datos trimestrales } \\
\hline \multicolumn{2}{c}{${ }_{\text {LnISR }}=c_{2}+\rho_{2} E C_{t-1}+\sum_{i=1}^{p} \gamma_{i} \Delta L n P L_{t-i}+\sum_{i=1}^{p} \theta_{i} \Delta L n I S R_{t-1}+U_{t}$} \\
\hline Número de rezagos & Estadístico $F$ & Probabilidad \\
\hline 1 & 3.0902 & 0.0531 \\
2 & 3.0776 & 0.0348 \\
3 & 4.1201 & 0.0055 \\
4 & 3.1536 & 0.0146 \\
\hline
\end{tabular}

Fuente: elaboración propia.

\section{CONCLUSIONES}

Dependiendo de dónde se ubique la fuerza conductora y causal, si es en el salario real o en la productividad laboral, muy diferentes terminan siendo las ideas con que se analizan y diseñan las políticas dirigidas al mercado de trabajo. Sin la menor duda, un enfoque donde prevalezca la idea de que la causalidad va desde la productividad hasta los salarios, recurrirá a un sistema donde los incrementos salariales estén regulados por el rumbo que tome la productividad. Bajo esta perspectiva es permisible entonces que las unidades productivas, donde incluso habitan trabajadores insatisfechos, no suban los salarios, mientras no aumente la productividad, ya que de lo contrario el incremento de los costos unitarios deteriorará la competitividad. Pero si entre un buen número de variables, el salario real constituye, por los distintos canales ya comentados, una fuente que provoca cambios en la productividad laboral, los temores arrojados por la lógica anterior serán infundados.

Los resultados de este estudio para datos anuales y trimestrales de la economía venezolana indican, al igual que otros estudios para países desarrollados y en desarrollo, que la causalidad va desde los salarios reales hasta la productividad del trabajo. En consecuencia, prescripciones que suelen imponer restricciones al incremento de los salarios reales atándolos a incrementos en la productividad deben ser vistos con cautela, por decir lo menos. 


\section{BIBLIOGRAFÍA}

Akerlof, G. (1982). Labor contracts as partial gift exchange. Quarterly Journal of Economics, 97(4). https://doi.org/10.2307/1885099

Banco Interamericano de Desarrollo (BID) (2010). La era de la productividad: cómo transformar las economías desde sus cimientos. Banco Interamericano de Desarrollo.

Baptista, A. (2005). El capitalismo rentístico: elementos cuantitativos de la economía venezolana. Cuadernos del CENDES, 22(60). http://ve.scielo.org/ scielo.php?script $=$ sci_arttext\&pid=S1012-25082005000300005

Brida, J. G., Risso, W. A. y Sanchez Carrera, E. J. (2010). Real wages as determinant of labour productivity in the Mexican tourism sector. European Journal of Tourism Research, 3(1). https://ssrn.com/abstract $=1308577$

Clark, J. B. (1899). The distribution of wealth: A theory of wages, interest and profits. Macmillan.

Engle, R. F. y Granger, C. W. (1987). Cointegration and error correction: Representation, estimation, and testing. Econometrica, 55(2). https://doi. org/10.2307/1913236

Ferguson, C. y Gould, J. (1980). Teoría microeconómica. Fondo de Cultura Económica.

Fondo Monetario Internacional (FMI) (2017). World Economic Outlook, seeking sustainable growth: Short-term recovery, long-term challenges. Washington, DC, October. http://dx.doi.org/10.5089/9781484312490.081

Gordon, D. M. (1994). Bosses of different stripes: A cross-national perspective on monitoring and supervision. The American Economic Review, 84(2). https://www.jstor.org/stable/2117862

Gordon, R. (1987). Productivity, wages, and prices inside and outside of manufacturing in the U.S., Japan, and Europe. European Economic Review, 31(3). https://doi.org/10.1016/0014-2921(87)90089-4

Granger, C. W. J. (1969). Investigating causal relation by econometric and crosssectional method. Econometrica, 37(5). https://doi.org/10.2307/1912791

Green, R. K. (1997). Follow the leader: How changes in residential and non-residential investment predict changes in GDP. Real Estate Economics, 25(2). https://doi.org/10.1111/1540-6229.00714

Hatanaka, M. (1996). Time-series-based econometrics. Oxford University Press. Hein, E. y Tarassow. A. (2009). Distribution, aggregate demand and productivity growth: Theory and empirical results for six OECD countries based on a post-Kaleckian model. Cambridge Journal of Economics, 34(4). 10.1093/ cje/bep066 
Hossain, A. (2005). The Granger-causality between money growth, inflation, currency devaluation and economic growth in Indonesia: 1954-2002. International Journal of Applied Econometrics and Quantitative Studies, 2(3). https://www.usc.gal/economet/reviews/ijaeqs234.pdf

Islam, R., Kiyondo, A. y Nganga, J. (2015). Real wages and labor productivity in Tanzania: How do they link? Journal of African Studies and Development, 7(3). http://hdl.handle.net/20.500.11810/4656

Kaldor, N. (1961). Capital accumulation and economic growth. En F. Lutz, F. Hague (eds.). The theory of capital. St Martin's Press.

Karaalp-Orhan, H. (2017). Labour productivity, real wages and unemployment: An application of bounds test approach for Turkey. Journal of Economic and Social Development, 4(2). http://www.jesd-online.com/dokumenti/upload/separated/Vol\%204\%20No\%202_Paper2.pdf

Kumar, S., Webber, D. y Perry, G. (2012). Real wages, inflation and labour productivity in Australia. Applied Economics, 22(23). https://doi.org/10.1 080/00036846.2011.568405

Lucidi, F. (2008). Labour market flexibility and productivity growth: New evidence from firm-level data. Preliminary Working Paper. "Sapienza" Università di Roma and Fondazione Giacomo Brodolini, Roma. http:// www.aiel.it/Old/bacheca/BRESCIA/papers/lucidi.pdf

Madsen, J. y Damania, R. (2001). Labour demand and wage-induced innovations: Evidence from the OECD countries. International Review of Applied Economics, 15(3). https://doi.org/10.1080/02692170110052365

Marquetti, A. (2004). Do rising real wages increase the rate of labor-saving technical change? Some econometric evidence. Metroeconomica, 55(4). https://doi.org/10.1111/j.1467-999X.2004.00201.x

Marx, K. (1967). Capital, vol. I. International Publishers. (Original publicado en 1867).

Méndez, J. y Hernández, H. (2014). Relación de largo plazo y análisis de causalidad y sensibilidad entre los salarios reales y la productividad laboral en el sector manufacturero a partir de cifras de los departamentos en Colombia. Revista Finanzas y Política Económica, 6(2). http://www.scielo.org. co/pdf/fype/v6n2/v6n2a06.pdf

Nayaran, P. y Smyth, R. (2009). The effect of inflation and real wages on productivity: New evidence from a panel of G7 countries. Applied Economics, 41(10). https://doi.org/10.1080/00036840701537810

Organisation for Economic Co-operation and Development (OECD) (2015). The labour share in G20 economies, report prepared for the G20 employment working group, antalya, Turkey. https://www.oecd.org/g20/ 
topics/employment-and-social-policy/The-Labour-Share-in-G20-Economies.pdf

Pyo, H. (2018). Labor productivity and a test of Kaldor-Verdoorn law in East Asia. En D. K. Das (eds.). Productivity dynamics in emerging and industrialized countries. Routledge India. 10.4324/9781351002547-8

Ricardo, D. (2005). Principles of political economy and taxation. Barnes \& Noble Books. (Original publicado en 1817).

Schmookler, J. (1966). Invention and economic growth. Harvard University Press.

Sims, C. A. (1972). Money, income and causality. American Economic Review, 62(4). https://www.jstor.org/stable/1806097

Smith, A. (1776). An inquiry into the nature and causes of the wealth of nations, vols. I y II. Oxford University Press.

Soo Khoon, G. (2009). Is productivity linked to wages? An empirical investigation in Malaysia. CenPRIS Working Paper Series 102/09. https://mpra. ub.uni-muenchen.de/18095/1/MPRA_paper_18095.pdf

Storm, S. y Naastepad, C. W. N. (2007). Why labour market regulation may pay off: Worker motivation, co-ordination and productivity growth. International Labour Organization, Economic and Labour Market Paper 2007/4. https://www.ilo.org/empelm/pubs/WCMS_113903/lang--en/in dex.htm

y Naastepad, C. W. N. (2009). Labor market regulation and productivity growth: Evidence for twenty OECD countries (1984-2004). Industrial Relations: A Journal of Economy and Society, 48(4). https://doi. org/10.1111/j.1468-232X.2009.00579.x

Strauss, J. y Wohar, M. (2004). The linkage between prices, wages and labor productivity: A panel study of manufacturing industries. Southern Economic Journal, 70(4). https://doi.org/10.2307/4135280

Sylos-Labini, P. (1983). Factors affecting changes in productivity. Journal of Post Keynesian Economics, 6(2). https://www.jstor.org/stable/4537805

Upender, M. y Sujan, M. (2008). Cointegration between labor productivity and wage rates: Empirical evidence from the Indian industries. The ICFAI Journal of Industrial Economics, 5(1). https://iupindia.in/208/IJIE_Cointegration_Labor_Productivity_Wage_Rates_39.html

Valadkhani, A. (2003). An empirical analysis of Australian labour productivity. Australian Economic Papers, 42(3). 10.1111/1467-8454.00199

Veerger, R. y Kleinknecht, A. (2011). The impact of labor market deregulation on productivity: a panel data analysis of 19 OECD countries. Journal of Post Keynesian Economics, 33(2). 10.2307/23035719 
y Kleinknecht, A. (2014). Do labour market reforms reduce labour productivity growth? A panel data analysis of 20 oECD countries (19602004). International Labor Review, 153(3). https://doi.org/10.1111/ j.1564-913X.2014.00209.x

Weisskopf, T., Bowles, S. y Gordon, D. (1983). Hearts and minds: A social model of U.S. productivity growth. Brooking Papers on Economic Activity, 14(2). https://doi.org/10.2307/2534294

Wooldridge, J. (2013). Introductory econometrics: A modern approach. SouthWestern Cengage Learning.

Yildrim, Z. (2015). Relationships among labour productivity, real wages and inflation in Turkey. Economic Research-Ekonomska Istraživanja, 28(1). 10.1080/1331677X.2015.1022401

Yusof, S. (2008). The long-run and dynamic behaviors of wages, productivity and employment in Malaysia. Journal of Economic Studies, 35(3). https:// doi.org/10.1108/01443580810887805 
